\title{
A novel human interleukin-24 peptide created by computer-guided design contributes to suppression of proliferation in esophageal squamous cell carcinoma Eca-109 cells
}

\author{
QUNFENG MA $^{1 *}$, XUEFENG DENG $^{1 *}$, BANGMING JIN $^{2,3}$, YAO ZHANG $^{2}$, \\ DAN LUO ${ }^{2}$, HEYU SONG $^{2}$, PENGKUN WANG $^{2}, \mathrm{CHI} \mathrm{ZHANG}^{2}, \mathrm{XUE} \mathrm{LI}^{2}, \mathrm{YINAN} \mathrm{SHI}^{2}$, YAN LIU $^{3}$, \\ ZHINAN $\mathrm{CHEN}^{4}$, ZILING WANG ${ }^{2}$ and $\mathrm{HONG} \mathrm{JIANG}^{2}$
}

\begin{abstract}
${ }^{1}$ Department of Thoracic Surgery, Affiliated Hospital of the Academy of Military Medical Sciences, Fengtai, Beijing 100071;
${ }^{2}$ College of Life Science and Bioengineering, School of Science, Beijing Jiaotong University, Haidian, Beijing 100044;

${ }^{3}$ College of Life Science, Southwest University, Beibei, Chongqing 400715; ${ }^{4}$ Cell Engineering Research Center,

The Fourth Military Medical University, Xicheng, Xi'an, Shaanxi 710032, P.R. China
\end{abstract}

Received July 10, 2014; Accepted October 17, 2014

DOI: $10.3892 / o r .2014 .3589$

\begin{abstract}
Based on the three-dimensional modeling structure of human interleukin-24 (hIL-24) and its most likely active position predicted by solvent accessibility and apparent electrostatic properties, a novel hIL-24 peptide M1 was created by computer-guided molecular design. The cytotoxicity and cell selectivity of M1 were examined in three human carcinoma cell lines and one normal human embryo lung fibroblast cell line (HEL). MTT assay showed that M1 induced growth arrest in two IL-20 receptor complex-positive cancer cell lines (the esophageal squamous cell carcinoma cell line Eca-109 and the melanoma cell line A375), and antibodies against IL-24 or IL-20 receptor complexes significantly neutralized the inhibitory activity. Moreover, M1 had almost no cytotoxicity on the lung cancer A549 cell line, which lacks a full complement of the IL-20 receptor complexes, or on HEL cells that express the IL-20 receptor complexes. These findings demonstrate that M1 could act as an excellent candidate for the induction of growth arrest on receptor complex-positive cancer cells. In summary, the M1 peptide may represent a novel anticancer agent for esophageal squamous cell carcinoma therapy due to its cancer cell selectivity and its relatively low cytotoxicity to normal cells.
\end{abstract}

\footnotetext{
Correspondence to: Dr Hong Jiang or Dr Ziling Wang, College of Life Science and Bioengineering, School of Science, Beijing Jiaotong University, No. 3 Shangyuancun, Haidian, Beijing 100044, P.R. China E-mail: jhong@bjtu.edu.cn

E-mail: zlw@bjtu.edu.cn

*Contributed equally
}

Key words: human interleukin-24, computer-guided molecular design, esophageal squamous cell carcinoma Eca-109 cell line, peptide

\section{Introduction}

Despite a better understanding of tumor cell biology (1), the treatment of most cancers has not significantly changed in the past three decades, and drugs that do not discriminate between tumor cells and normal tissues remain the mainstay of anticancer therapy (2). Therefore, finding anticancer drugs that specifically kill tumor cells with no obvious effects on normal cells is the research initiative around the world. Notably, in 1995, Jiang et al (3) reported that melanoma differentiation associated gene-7/interleukin-24 (mda-7/IL-24) inhibited the proliferation of melanoma cells. IL-24 is an intriguing and exciting molecule due to its ability to selectively kill and induce apoptosis in a wide range of cancer cells, both in vitro and in vivo, without harming the equivalent normal human cells, including epithelial cells, fibroblasts, melanocytes and orastrocytes $(4,5)$. This characteristic has led to its characterization as a 'tumor suppressor' (6). Of importance, IL-24 also exerts immunomodulatory effects $(7,8)$, and combinatorial treatment with other conventional therapeutic modalities or agents, such as radiotherapy $(4,9,10)$, chemotherapy $(11,12)$, monoclonal antibody therapy $(4,13)$, sulindac $(4)$, antisenseoligonucleotides (14), reactive oxygen species generators (15), and non-steroidal anti-inflammatory drugs (NSAIDs) (16), can induce dramatic enhancement in its apoptotic and tumorsuppressing capacities compared to its use as a single agent $(17,18)$. Dr Paul B. Fisher, its discoverer, suggested that this novel cytokine may be the long sought after and proverbial 'magical bullet' for a diverse set of cancers (19).

Ectopic expression of IL-24 in cancer cells, either by transfection of the tumor cells with a plasmid containing IL-24 cDNA (20) or by infecting the tumor cells with a recombinant adenovirus (Ad.IL-24) (21) or adeno-associated virus (AAVIL-24) (22) to deliver the gene, rapidly inhibits the growth of cells and induces apoptosis in a broad spectrum of human and rodent tumors in vitro and in vivo. In contrast, overexpression of IL-24 in normal primary or immortalized cells does not affect 
their growth and viability. The overexpressed IL-24 protein was shown to localize to the endoplasmic reticulum (ER)/ Golgi compartments and was secreted from infected tumor cells as soluble IL-24 (sIL-24), resulting in 'toxic bystander' effects on distant uninfected tumor cells $(5,23)$. This effect is expected for a cytokine and could make IL-24 treatment an ideal and effective tool for cancer gene therapy. The exogenous IL-24 protein can robustly induce the expression of endogenous IL-24, which generates the signaling events necessary for 'toxic bystander' killing and is essential for recombinant IL-24-induced apoptotic effects (24).

The recombinant IL-24 protein can act on tumor cells in different ways. Bacterially synthesized GST-IL-24 can be taken up by cells (25), or its entry into tumor cells is mediated in a receptor-independent manner. Yet, some researchers did not observe any apoptosis-inducing effects of GST-IL-24 or other bacterially expressed IL-24 on melanoma and lung cancer cells (25). However, the purified recombinant IL-24 protein, synthesized in mammalian cells and secreted into the cell media, preferred to kill cancer cells in a receptor-dependent fashion. IL-24 binds to the currently recognized IL-20 receptor complexes; these complexes consist of two sets of heterodimeric chains, IL-20R1/IL-20R2 or IL-22R1/IL-20R2 (25-27). Upon ligand binding, both receptors induce the phosphorylation of STAT3, which then translocates into the nucleus where it upregulates Bax and induces apoptosis in melanoma cells (26). In contrast to GST-IL-24 and Ad.IL-24, purified IL-24 synthesized in mammalian cells does not appear to have any biological effect on cells lacking expression of the IL-20 receptor complexes, such as the human lung cancer A549 cell line in vitro (6).

Even though full-length IL-24 cDNA has been used in most experiments, early results showed that a specific peptide of IL-24 (M4, residues 104-206) mimics the biological properties of the full-length protein, including its in vivo tumor suppression properties. M4 interacted with the endoplasmic reticulum chaperone $\mathrm{BiP} / \mathrm{GRP} 78$ through the conserved sequences in its $\alpha$-helices $\mathrm{C}$ and F; M4 also maximally activated p38 MAPK and promoted the expression of its downstream targets, such as GADD34 and GADD153 (28). At the same time, bacterially synthesized GST-M4 has a higher expression level than GST-IL-24 because of its smaller size, but GST-M4 is also equally as potent as GST-IL-24 at inhibiting growth and inducing apoptosis in four NSCLC cell lines. At low doses, GST-M4 was relatively more potent than GST-IL-24 in exerting its killing effect. Both GST-IL-24 and GST-M4 activated the p38 MAPK pathway, which is involved in mediating the induction of apoptosis by IL-24 protein in several tumor models (29). Thus, small-molecule peptides that mimic the IL-24 protein may lead to new therapeutics that can selectively target and kill cancer cells based on their increased level of stress compared with normal cells. At present, it is not clear whether an IL-24 peptide smaller than M4 could have the same activity as the full length IL-24.

The use of peptides in cancer therapeutics has recently become popular because of their potency, specificity, low toxicity, and the limitations of viral vector gene therapy approaches $(30,31)$. Small anticancer peptides can specifically inhibit the proliferation of cancer cells during cancer development and have beneficial effects on multidrug resistant cancer cells due to their low molecular weight and high efficiency. At the same time, small anticancer peptides can be administered to patients in many forms and do not induce immune reactions. Compared to large recombinant protein drugs, peptides are easier to be restructured and synthesized at low cost. All of these properties show that small anticancer peptides have the potential to be widely applied as clinical anticancer treatments.

Assuming that the M4 mutant has the same bioactivity against cancer cells as the full-length protein, we hypothesized that IL-24 peptides smaller than M4 may inhibit the proliferation of cancer cell lines. To test this hypothesis, a small peptide, M1, was designed using a computer-guided modeling method, and we carefully assessed the effect of this peptide on the proliferation of cancer cells in vitro. Our results revealed that the chemically synthesized M1 peptide specifically inhibited the growth of the esophageal squamous cell carcinoma (ESCC) cell line Eca-109 and the melanoma cell line A375, while having almost no effects on the human embryo lung (HEL) fibroblast cell line. The aim of the present study was to identify the therapeutic potential of a small IL-24 peptide that can selectively target and kill cancer cells.

\section{Materials and methods}

Cells and materials. The melanoma carcinoma cell line A375 and the lung cancer cell line A549 were obtained from the Cell Center of Peking Union Medical College. The ESCC cell line Eca-109 and the human embryo lung fibroblast cell line HEL were kindly provided by Dr Xiaofei Zheng at the Beijing Institute of Radiation Medicine. All cells were checked by STR (short tandom repeat) analysis in Beijing Microread Biotechnology Co. and maintained in Dulbecco's modified Eagle's medium (DMEM; Gibco, Inc., Logan, UT, USA) supplemented with $10 \%$ fetal bovine serum (FBS; Life Technologies, Inc., Grand Island, NY, USA), 100 units/ml penicillin, $100 \mu \mathrm{g} / \mathrm{ml}$ streptomycin, $2 \mathrm{mmol} / \mathrm{l} \mathrm{L}$-glutamine, and HEPES buffer (Life Technologies). Anti-IL-24 antibodies (AF1965 and MAB1965) and anti-IL-20R1 (MAB11762), anti-IL-20R2 (AF1788) and anti-IL-22R1 (MAB2770) antibodies were purchased from R\&D Systems China Co., Ltd. (Shanghai, China). All other primary and secondary antibodies were purchased from Beijing Zhongshan Golden Bridge Biotechnology Co. (Beijing, China).

\section{Computer modeling design and chemical synthesis of the IL-24 peptide}

Theoretical simulation of the space structure of IL-24. First, we retrieved the sequence information of IL-24 from the UniProtKB/SwissProt database (http://www.ebi.ac.uk). Then, with the help of the BLastP program (http://www.ncbi.nlm. nih.gov/BlastP), we made a similar retrieval from the Protein Data Bank, where we obtained the protein with the highest sequence similarity to IL-24. Finally, using computer guided homologous modeling technology and with the aid of the Homology module of the InsightII 2005 package, we modeled a three-dimensional theoretical structure of hIL-24.

\section{Analysis of the physical and chemical properties and apparent characteristics of IL-24 based on its spatial structure. We analyzed the solvent accessibility of IL-24 based on its spatial modeling structure and the solvent accessibility principle. We}


A

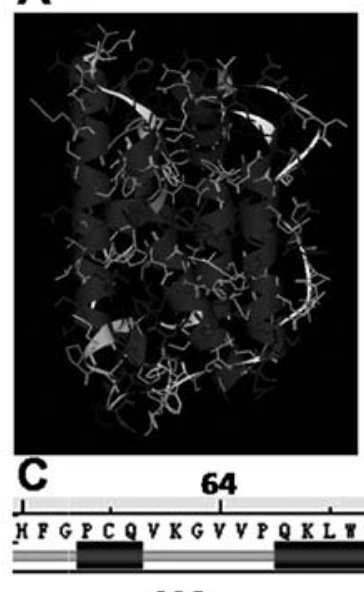

B

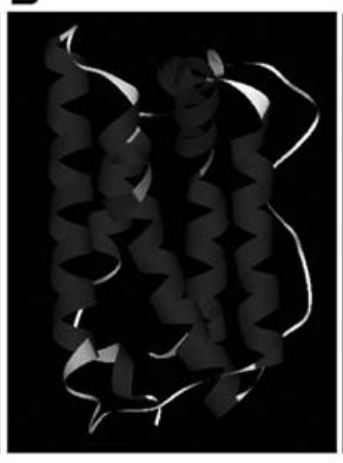

74
D

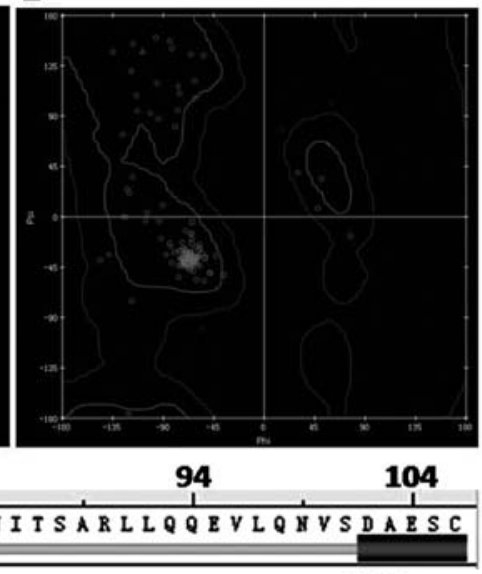

154

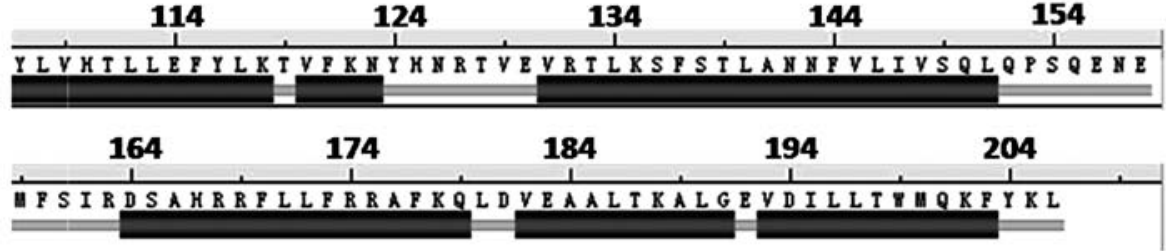

Figure 1. Simulation of the three-dimensional structure of hIL-24. (A and B) The space structure of hIL-24 obtained by homologous modeling and the mechanics optimization method. (C) The predicted secondary structure modeling based on the K-S rules. (D) The space structure of IL-24 using the R-diagram.

also analyzed the surface electrostatic potential distribution of IL-24 using the Delphi program.

Prediction of potential active sites in the IL-24 space structure. Using our reasonable IL-24 space structure, combined with the predicted IL-24 apparent physical and chemical properties (solvent accessibility and apparent electrostatic properties), we predicted the most likely active position of IL-24 based on the prediction method of the binding site.

Chemical synthesis of $I L-24$. The peptides used in the present study were synthesized by Beijing B\&M Biotech Co., Ltd. (Beijing, China). All peptides were synthesized by solid-phase chemistry, purified to homogeneity ( $>90 \%$ purity) by reversedphase high-pressure liquid chromatography (RP-HPLC), and assessed by mass spectrometry (MS). The peptides were dissolved in DMEM.

Immunofluorescence staining and fluorescence microscopy. Briefly, $5 \times 10^{4}$ cells were seeded in a 96-well plate, maintained in DMEM with $10 \%$ FBS and cultivated for 24 h to $60-70 \%$ confluency at $37^{\circ} \mathrm{C}$. The cells were washed with PBS three times, fixed with $4 \%$ formaldehyde, permeabilized with $0.5 \%$ NP40 in PBS, and blocked with 1\% BSA in PBS for $30 \mathrm{~min}$ at room temperature. Then, the cells were incubated with anti-IL-20R1, anti-IL-20R2 or anti-IL-22R1 (1:10) antibody in PBS overnight at $4^{\circ} \mathrm{C}$. The cells were washed and incubated in TRITC-conjugated secondary antibody (1:200) in PBS for $1 \mathrm{~h}$ at room temperature. The nuclei were stained using DAPI and examined by fluorescence microscopy (Nikon, Tokyo, Japan), and the resulting images were merged using fluorescence microscopy (original magnification, x200). Controls were incubated with only the secondary antibodies under the same experimental conditions.
Cell proliferation assay. Cell viability was assessed by MTT assays as previously described (15). Briefly, cells were seeded in 96-well plates at a density of 2,500 cells/well for $24 \mathrm{~h}$ at $37^{\circ} \mathrm{C}$. The next day, the cells were treated with the peptide M1 at different concentrations of $0.5,1,2,5,10,20,50,100$ or $200 \mu \mathrm{g} / \mathrm{ml}$. On day 4 after treatment, the medium was removed, and MTT (final concentration of $0.5 \mathrm{mg} / \mathrm{ml}$ ) was added to each well. The cells were maintained at $37^{\circ} \mathrm{C}$ for $4 \mathrm{~h}$, and then $150 \mu \mathrm{l}$ DMSO was added to each well and mixed thoroughly. The absorbance from the plates was read on a Bio-Rad microplate reader Model 550 at $490 \mathrm{~nm}$. MTT absorbance of the untreated control cells was set to 1 to calculate the relative number of viable cells. The experiments were repeated at least three times to ensure reproducibility and statistical significance.

Statistical analysis. All of the experiments were performed at least three times. Statistical comparisons between the groups were performed using an unpaired Student's t-test with the SPSS version 15 software (SPSS, Inc., Chicago, IL, USA). $\mathrm{P}<0.05$ was considered to indicate a statistically significant result, and $\mathrm{P}<0.01$ was considered to indicate a highly statistically significant result.

\section{Results}

Theoretical simulation of the space structure of $I L-24$. We used the UniProtKB/SwissProt (http://www.ebi.ac.uk) database to retrieve the protein sequence of human IL-24 (UniProtKB code: Q13007). hIL-24 is composed of 206 amino acids, where 1-51 represent the signal peptide, and 52-206 represent the mature extracellular chain. N-glycosylation occurs at sites 85,99 and 126 .

Using computer guided homologous modeling technology and the InsightII 2005 package with the Homology module, we 
constructed a three-dimensional structure of hIL-24. Selecting CVFF (consistent valence force field), the CHARMM force field in turn, the steepest descent (convergence criterion, $0.02 \mathrm{Kcal} / \mathrm{mol}$; convergence step: 200,000 steps), and the conjugate gradient (convergence criterion, $0.01 \mathrm{Kcal} / \mathrm{mol}$; convergence step, 250,000 steps) methods were used to optimize the initial spatial conformation of hIL-24. The optimized conformation of hIL-24 is shown in Fig. 1A and B. The hIL-24 possesses structural properties that are found in the cytokine superfamily, including four long anti-parallel helical structures connected by a random coil (Fig. 1C, helix A, 67-82; helix B, 102-123; helix C, 131-151; helix D, 164-203). The secondary structure of hIL-24 was analyzed based on the K-S rules and the secondary structure of IL-24 is shown in Fig. 1C. Making use of the spatial modeling structure of hIL-24, we rationally assessed its structure using R-diagram. As shown in Fig. 1D, the spatial conformation of the hIL-24 model is rational. Therefore, the selected simulation optimization and the conformation optimization force field and the methods we used were all adequate.

Analysis of the physical and chemical properties and apparent characteristics based on the spatial structure of $I L-24$. Space structure and the apparent characteristics of a protein are related closely to its biological function. We analyzed the solvent accessibility of IL-24 based on the spatial structure of the protein and the solvent accessibility principle. The solvent accessibility of IL-24 is shown in Fig. 2A; blue amino acid residues are fully exposed to the solution, while green amino acid residues are buried within the protein. Residues that are completely exposed to the solvent, such as Loop AB (83-104) or Loop CD (154-164), may constitute potential functional sites of IL-24.

We analyzed the surface electrostatic potential distribution of IL-24 using the Delphi program. As shown in Fig. 2B, the blue regions represent positive electricity areas, the red regions represent negative electricity areas, and the white regions are neutrally charged. The N-terminus of Loop $\mathrm{AB}$ and the $\mathrm{C}$-terminus of Loop $\mathrm{CD}$ have strong negative electricity, while the C-terminus of Loop AB and the N-terminus of Loop CD have strong positive electricity, suggesting that these sites may be potential functional sites.

Prediction of potential active sites in the IL-24 space structure. Based on the IL-24 space structure which was reasonably simulated and combined with the predicted IL-24 physical and chemical properties (solvent accessibility and apparent electrostatic properties), we defined the globe core reasonably and predicted the most likely active position using the prediction method of the binding site. As shown in Fig. 2C-H, the yellow ball represents the defining core position, while the green point represents a potential active site. Within the IL-24 potential active sites, sites $\mathrm{G}$ and $\mathrm{H}$ generally cannot become active since they include the amino acid residues within the screw. The rest of the sites, such as C, D, E and $\mathrm{F}$, are residues in the Loop $\mathrm{AB}$ or Loop $\mathrm{CD}$; in addition, the $\mathrm{N}$-terminus and $\mathrm{C}$-terminus of the Loop $\mathrm{AB}$ and the $\mathrm{N}$-terminus of Loop CD comprise the active site with the greatest potential. Thus, the peptide M1 we designed was 30 amino acid residues from 76 to 105 and its sequence was
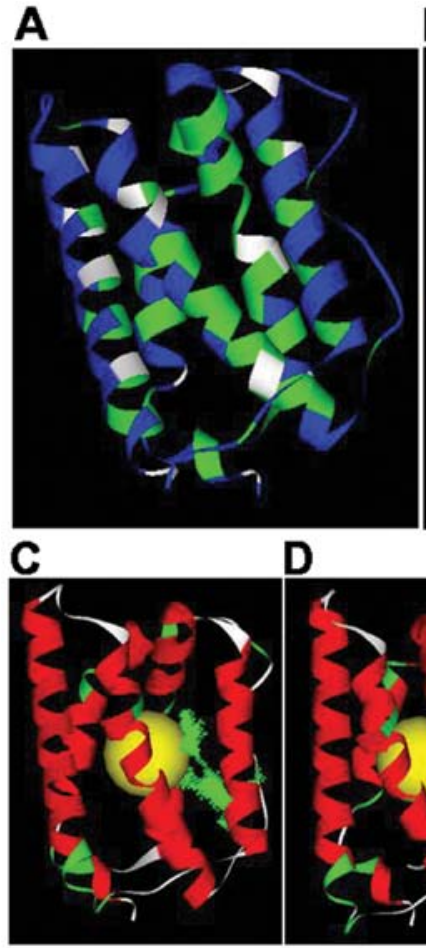

D
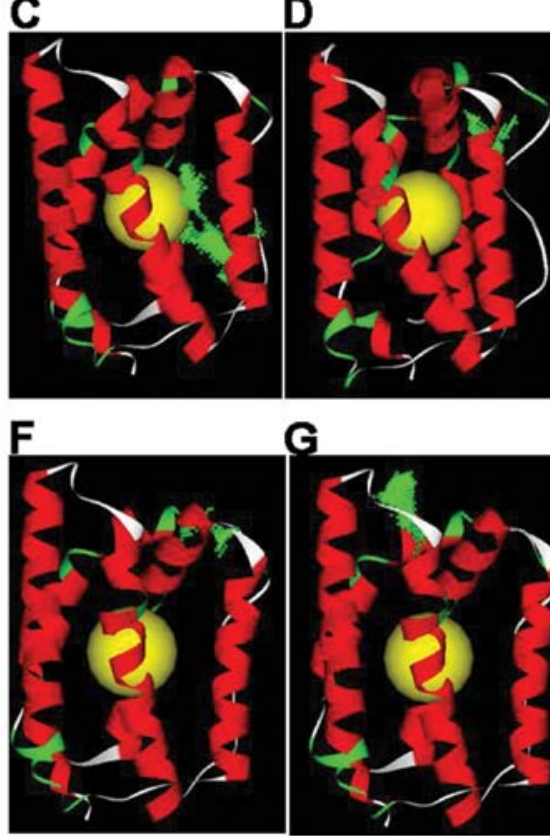

G

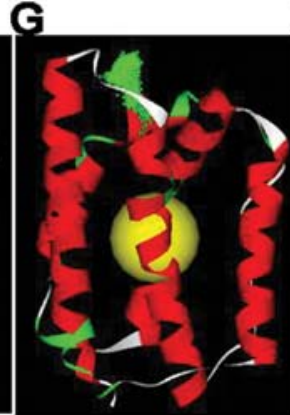

H

E
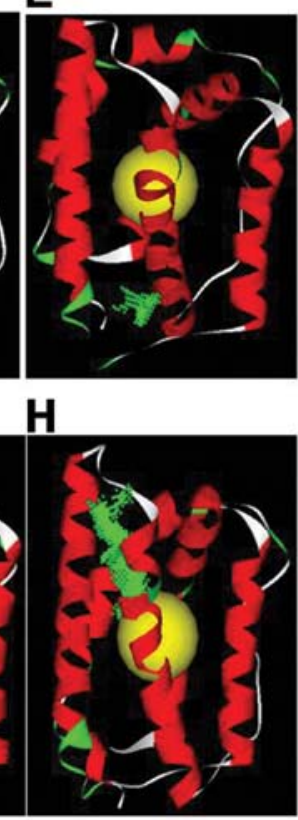

Figure 2. Predicted information for hIL-24. (A) The solvent accessibility of IL-24. (B) Apparent electrostatic distribution of IL-24. (C-H) Predicted active position based on the binding site.

VKDTMQAQDNITSARLLQQEVLQNVSDAES. We also designed another peptide M2 of IL-24 (residues 146-174, sequence was LIVSQLQPSQENEMFSIRDSAHRRFLLFR), which covered Loop $\mathrm{CD}$, and had nearly no function on proliferation of Eca-109 and A375 cells (data not shown).

Chemical synthesis of IL-24. The M1 peptides used in the present study were synthesized at the Beijing B\&M Biotech Co. using solid-phase chemistry and were purified to homogeneity ( $>95 \%$ purity) by RP-HPLC; the molecular weight of the peptide was determined by MS. As shown in Fig. 3, the purity of the M1 peptide was $98.46 \%$, while the molecular weight of M1 was $3333.4 \mathrm{Da}$, which is close to the theoretical value of $3332.68 \mathrm{Da}$. The peptides were dissolved in DMEM supplemented with $10 \%$ FBS.

Expression of IL-20 receptor complexes in the different cell lines. Classic cytokine signaling events require the binding of a cytokine to its receptor(s). The expression patterns of the IL-20 receptor complexes on the cell surface were identified by immunofluorescence staining and fluorescence 
A

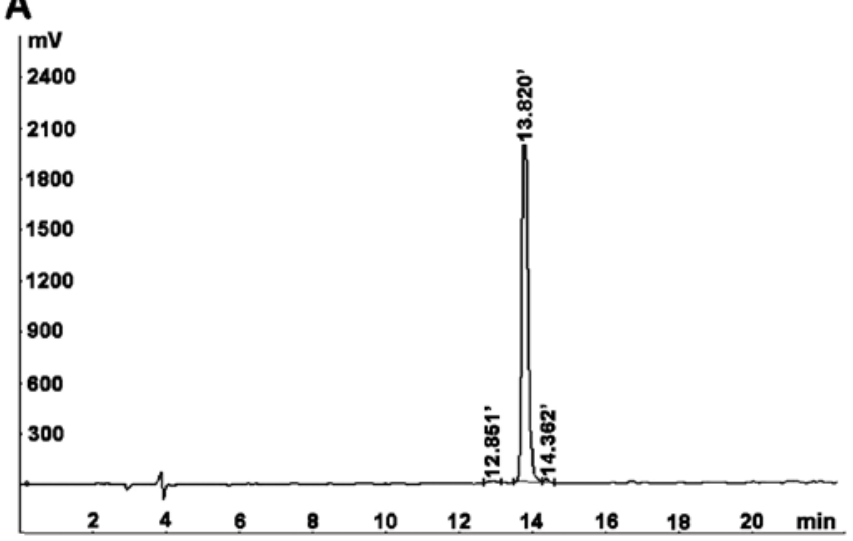

B

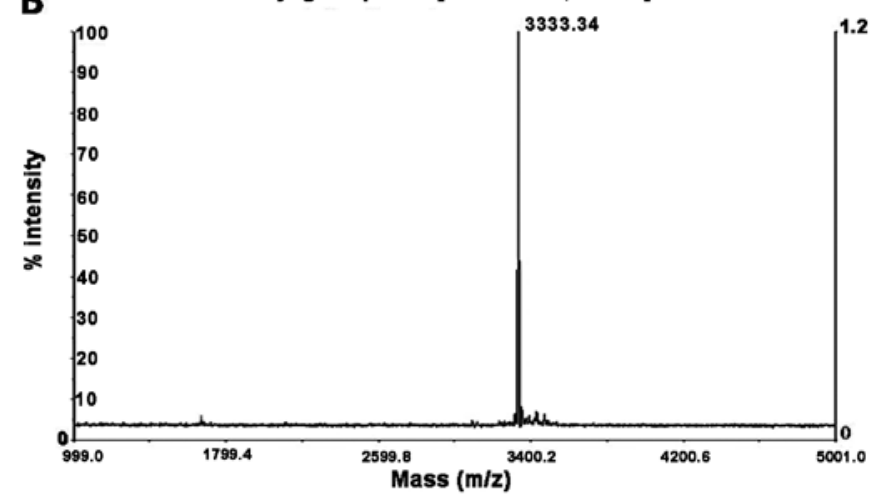

Figure 3. Results for the analysis of the IL-24 M1 peptide. (A) Purity analysis of the M1 peptide by high-pressure liquid chromatography (HPLC). (B) Mass spectrometry (MS) was used to detect the molecular weight of the M1 peptide.

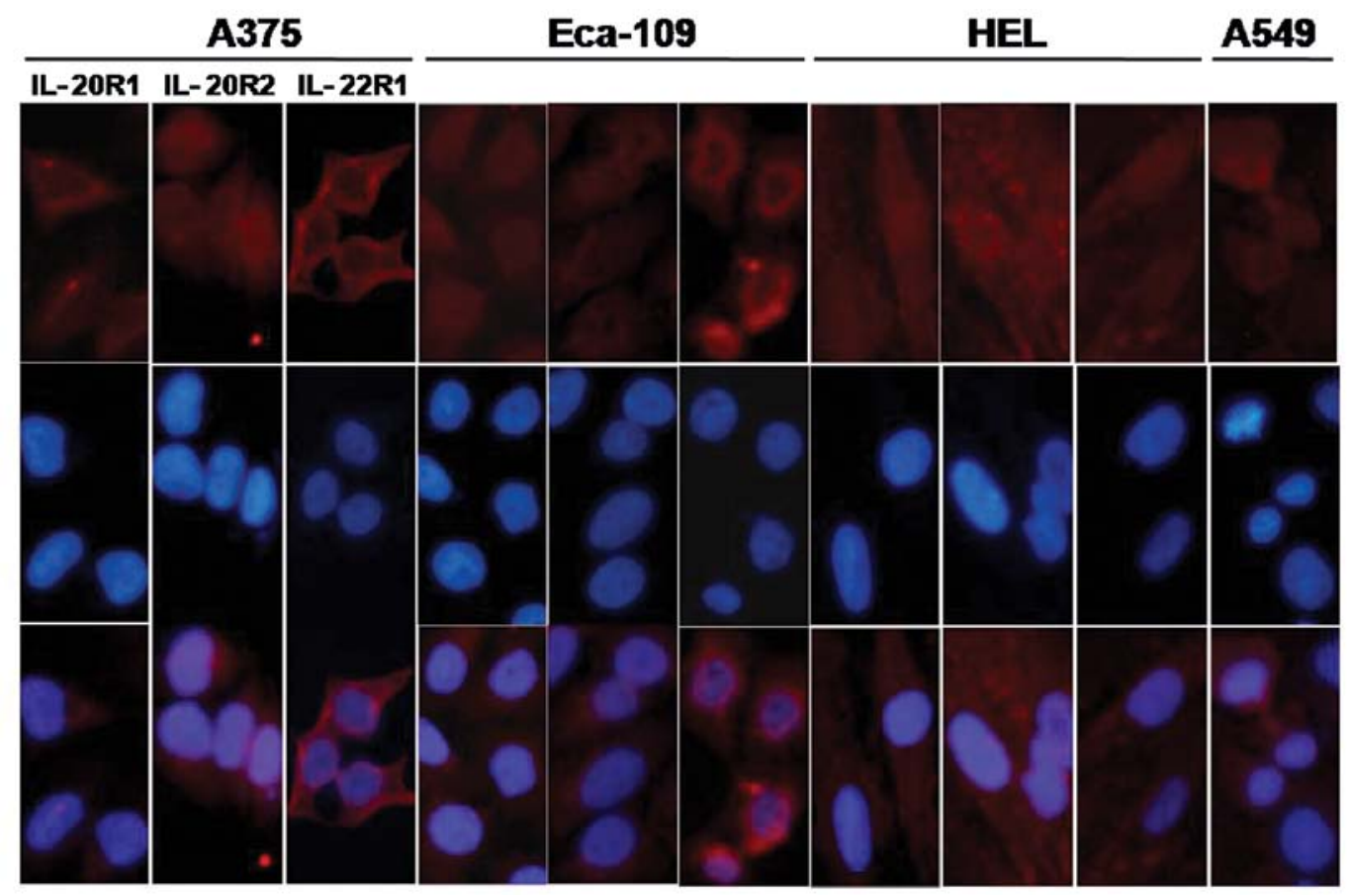

Figure 4. Expression patterns of the IL-20 receptor complexes analyzed by immunofluorescence. The Eca-109, A375 and HEL cell lines expressed three types of subunits, IL-20R1, IL-20R2 and IL-22R. The A549 cell line was positively stained for IL-20R2, and the two other receptor subunits were very weakly stained.

microscopy. As showed in Fig. 4, the Eca-109, A375 and HEL cell lines expressed three types of subunits, IL-20R1, IL-20R2 and IL-22R1. The A549 cell line was positively stained for IL-20R2, and the two other receptor subunits were not stained. Therefore, the A375 cells were used as a positive control for the expression of IL-20 receptor complexes, which are the target receptors of IL-24; A549 cells were used as the negative control for receptor expression, and HEL as the control of the primary normal cell line.

The M1 peptide selectively induces growth inhibition in the cancer cell lines. The effects of the M1 peptide on the growth of cells were analyzed 4 days post-treatment using the MTT assay. Different concentrations of the peptide, ranging from 0 to $200 \mu \mathrm{g} / \mathrm{ml}$, were tested and PBS was used as a control.
As shown in Fig. 5A, the M1 peptide induced a significant decrease $(\mathrm{P}<0.05 ; \mathrm{P}<0.01)$ in the cell viability of the Eca-109 and A375 cells, compared with the PBS-treated cells. In these two cell lines, a decrease in cell viability was evident even after treatment with 10 or $0.5 \mu \mathrm{g} / \mathrm{ml}$ of the M1 peptide, respectively and increasing concentrations of the peptide produced a more pronounced effect. The highest inhibition rate (\%) of the M1 peptide on the Eca-109 and A375 cell lines was $21 \%$ at $200 \mu \mathrm{g} / \mathrm{ml}$ and $27 \%$ at $2 \mu \mathrm{g} / \mathrm{ml}$, respectively. However, treatment with the M1 peptide at the same concentrations, $0.5-100 \mu \mathrm{g} / \mathrm{ml}$, did not produce a significant decrease in the viability of the other two cell lines (A549 and HEL); the M1 peptide had an effect on the HEL cell line only when it was administered at a very high concentration $(200 \mu \mathrm{g} / \mathrm{ml})$. To confirm that the inhibitory effect was due to treatment with 

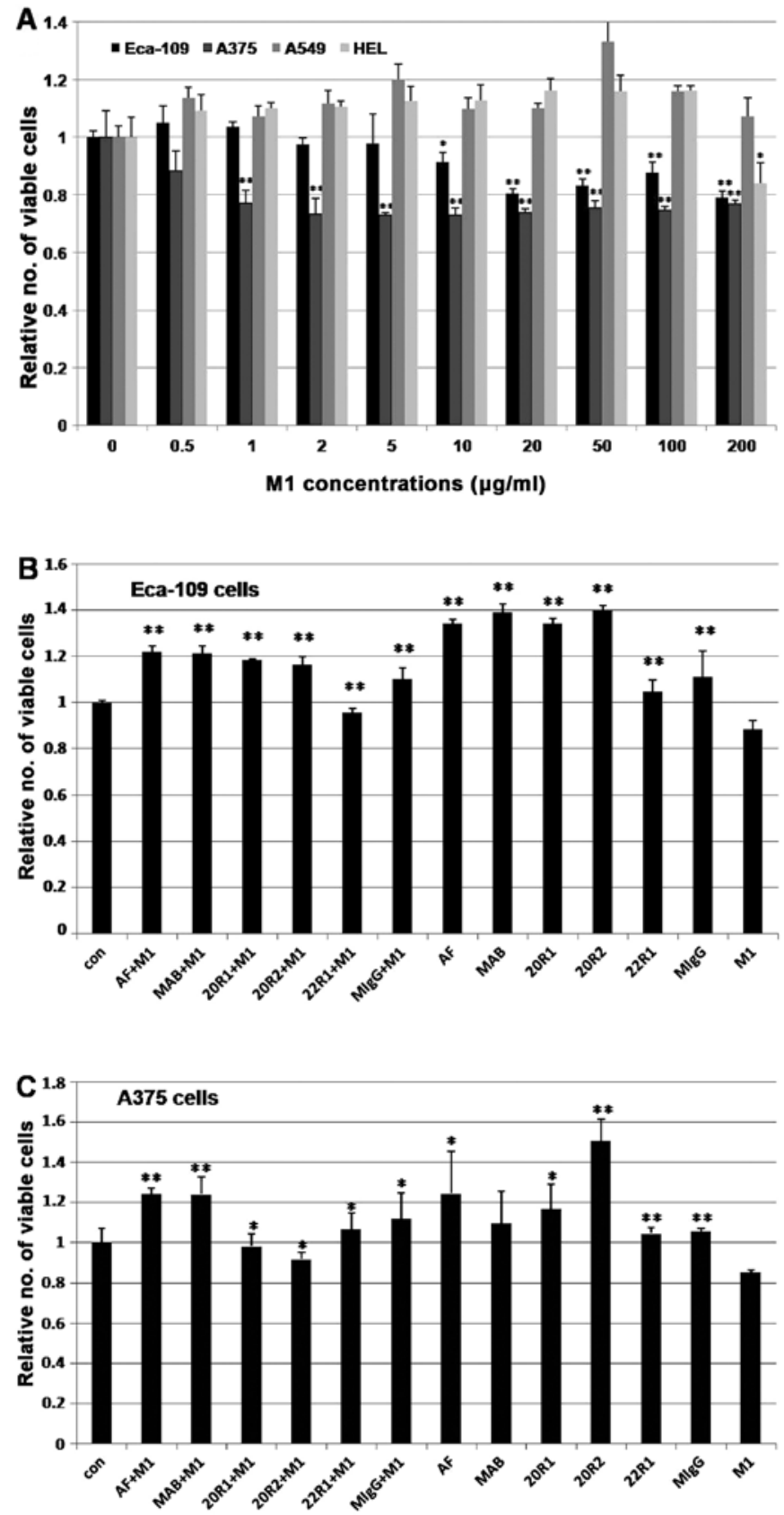

Figure 5. Comparative growth inhibition of the M1 peptide on different cells (A) The effect of the M1 peptide on cell viability. Cells (Eca-109, A375, A549 and HEL) were treated with the M1 peptide at different concentrations. A significant decrease in cell viability was observed in the Eca-109 and A375 cells, but almost no effects were observed in the A549 and HEL cells. (B and C) Inhibition of the proliferation of Eca-109 and A375 cells was significantly neutralized by the addition of anti-IL-24 antibodies (MAB, monoclonal antibody MAB1965; AF, polyclonal antibody AF1965) or anti-IL-20 receptor complex antibodies (20R1, anti-IL-20R1 antibody; 20R2, anti-IL-20R2 antibody; 22R1, anti-IL-22R1 antibody). Columns, the average of three independent experiments; bars, $\pm \mathrm{SD} .{ }^{*} \mathrm{P}<0.05 ;{ }^{* *} \mathrm{P}<0.01$

the M1 peptide, the IL-24 antibodies (polyclonal antibody AF1965 and monoclonal antibody MAB1965) and the IL-20R antibodies (polyclonal antibodies IL-20R1, IL-20R2 and IL-22R1) were added concurrently with the M1 peptide at a concentration of $100 \mu \mathrm{g} / \mathrm{ml}$. The inhibition of proliferation was significantly neutralized in the Eca-109 and A375 cell lines, as shown in Fig. 5B and C. These MTT results indicated that the M1 peptide can specifically inhibit the proliferation of cancer cell lines that express the IL-20 receptor complexes. Additionally, the M1 peptide had no biological effects on the growth or viability of cancer cell lines, such as A549, which lack expression of the IL-20 receptor complexes or primary normal cells that express the IL-20 receptor complexes.

\section{Discussion}

Since 1995, numerous studies have shown that IL-24 selectively kills a large variety of cancer cells, in vivo and in vitro, and leaves healthy cells unharmed. In a remarkably short time frame, IL-24 has changed from a laboratory discovery into a clinical treatment. These remarkable findings have led to the development of INGN 241, a replication-incompetent IL-24expressing adenovirus, which is currently being assessed in clinical trials (Introgen Company homepage) $(32,33)$. However, IL-24 therapy in its current form is a type of gene therapy, and based on simple mass action effects, it is not possible to infect every tumor cell within a tumor using an adenovirus even with intra-tumoral injection. This is one possible reason why so many gene therapy approaches have failed in the clinic (34).

To avoid the problems mentioned above, some alternative strategies have been developed. To develop these strategies, it is essential that the apoptosis-inducing properties and the mechanisms by which different recombinant forms of IL-24 protein (bacterial GST-fusion protein, baculovirus-expressed IL-24, secreted IL-24 from transfected mammalian cells) induce apoptosis are determined. In the present study, we demonstrated that peptide M1 created by computer-aided design can specifically inhibit the growth of the ESCC cell line Eca-109 and the melanoma cell line A375, but has almost no effects on human lung cancer A549 cells and the human embryo lung fibroblast cell line HEL. This result was identical to what we obtained with the full length IL-24 recombinant protein secreted from mammalian cells on these four types of cells (unpublished data).

Concerning the structure of IL-24, some studies have reported that IL-24 belongs to the 4-helix bundle family of cytokine molecules, which are most closely related to the IL-10 subfamily. Tertiary structure predictions, which are based on computer simulations, generated a compact globular structure consisting of 4 helical regions interspersed by loops of unpredicted structure $(4,35)$. However, there is no detailed information concerning these 4 helices. We created a three-dimensional structure of human IL-24 with the help of computer aided homologous modeling technology and the InsightII 2005 package with the Homology module. The IL-24 protein possesses structural properties that are always found in the cell factor superfamily, including four long antiparallel helical structures connected by a random coil. The four helices A, B, C and D in IL-24 include the 67-82, 102-123, 131-151 and 164-203 amino acids, respectively. On the other hand, some researchers have reported that all monomeric members of the IL-10 family can adopt similar patterns of tertiary folding, as predicted from the primary sequence alignment and computerbased threading analyses. All IL-10 family members have a consistent pattern of predicted $\alpha$-helical structure that corresponds to the known six-helices (A to F), based on the crystal 
structure of ebvIL-10, Epstein-Barr Virus-encoded IL-10 homolog $(36,37)$. In fact, the six-helices are the same as the four-helices since helices $\mathrm{C}$ and $\mathrm{D}$ can each be divided into two helixes.

Several experimental studies have shown that the IL-24 protein can be secreted from the cell, and purified IL-24 protein can interact with two different heterodimeric receptor complexes: type I (IL-20R1/IL-20R2) or type II (IL-22R1/ IL-20R2). In receptor-expressing cells, IL-24 activates the JAK-STAT pathway, as shown by phosphorylation of STAT3 $(33,38)$. The receptor chains IL-20R1 and IL-22R1 are widely expressed in many tissues; nevertheless, the expression of a functional IL-24 receptor depends on the presence of IL-20R2, the common chain for both receptor pairs, which is absolutely required for receptor activation (33). However, most of the IL-24 receptor expression studies relied on RT-PCR, which is neither quantitative nor does it establish the presence of the corresponding proteins and functional receptors. Therefore, we assessed the expression of the receptors using immunofluorescence staining. The Eca-109 and A375 cells expressed the complete set of IL-20 receptor complexes (IL-20R1, IL-20R2 and IL-22R1) and were efficiently treated by the M1 peptide. On the other hand, A549 cells lack a full complement of IL-20 receptor complexes, similar to the results of $\mathrm{Su}$ et al (10), which make the A549 cells relatively resistant to M1 peptide treatment. Our analysis revealed a variable level of IL-20 receptor complex expression in different cancer cell lines, indicating that an effective strategy needs to be utilized to facilitate the efficient infection of all types of cancer cells with the M1 peptide. Most studies show that A375 cells are positive for the expression of the IL-20 receptor complexes (39), but a few do not. Kreis et al (33) reported that A375 cells only express IL-22R1 according to RT-PCR and express no receptors according to western blot analysis; therefore, these cells did not react to the IL-24 protein as they do not express sufficient amounts of the specific receptor pairs. Many studies have reported that A549 cells do not express the IL-20 receptor complexes, and extracellular treatment with mammalian cell synthesized IL-24 resulted in no biological effect on cell growth or viability (6). However, when A549 cells were co-cultured with HEK293 cells producing sMDA-7/IL-24, the in vivo tumor growth of nude mice was inhibited. Similarly, systemic administration of sMDA-7/IL-24 also inhibited lung tumor growth in a mouse xenograft model. In this context, one would anticipate that IL-24 might exert its antitumor properties in vivo by evoking multiple pathways, including direct induction of cancer cell apoptosis, inhibition of angiogenesis and modulation of immune responses (4).

The morbidity of ESCC differs between countries or areas. China has the highest morbidity and the highest mortality of this disease. Approximately 310,400 new cases are diagnosed around the world each year, and $\sim 167,200$ of these cases are in China. According to the American Cancer Society (www. cancer.gov), in 2013, nearly 18,000 people in the United States were estimated to be diagnosed with ESCC, and 15,210 deaths were expected to occur from this disease. Pataer et al (23) reported that the adenoviral ER-targeted IL-24 vector (Ad-ER-IL-24) selectively and effectively inhibited the growth and proliferation of ESCC cells (Seg1 and Bic1) by enhancing cell death. Both Ad-IL-24 and Ad-ER-IL-24 activated a novel pathway of ER stress-induced apoptosis characterized by the unregulated expression of phosphorylated JNK (p-JNK), phosphorylated cJun (p-cJun), and phosphorylated RNA-dependent protein kinase (p-PKR). At the present time, it is not clear whether the IL-24 protein or peptide has biological functions in ESCC cells. Our previous results showed that the purified IL-24 secreted from transfected mammalian cells specifically inhibited proliferation in vivo and in vitro and could induce apoptosis in the ESCC cell line Eca-109 (unpublished data). The M1 peptide also inhibited the growth of Eca-109 cells. Our results indicate that Eca-109 cells are sensitive to IL-24 protein and peptide, and the use of the IL-24 peptide may be a new strategy for the treatment of ESCC.

Further studies are required to determine the effects of the M1 peptide on in vivo Eca-109 tumor models; if successful, these models could serve as an entry point for the translation of the treatment into the clinic setting by defining the safety and efficacy of the treatment in patients with ESCC. The antibodies against IL-24 (AF1965 and MAB1965) and IL-20 receptor subunits (IL-20R1, IL-20R2 and IL-22R1) could neutralize the inhibitive function of M1 on tumor cells, indicating that M1 may bind to the receptor and activate signaling in a receptor-dependent manner and that other cancer cells expressing IL-20 receptor complexes may be its target cells. It is not clear whether the M1 peptide would activate the receptor in the same way as full length IL-24 protein and whether some conventional therapeutic modalities or agents may strengthen the activity of M1 against cancer cells, as was shown for the full-length IL-24 protein and GST-M4. The range of cancer cells that might be affected by the M1 peptide remains to be identified. Although the M1 peptide can kill cancer cells, the half-life of the peptide needs to be improved by polyethyleneglycol (PEG) modification. Novel systemic delivery approaches, such as microbubbles or ultrasound delivery, are needed to enhance the administration of the M1 peptide, specifically to the tumor site or its microenvironment. It is also important to identify how the M1 peptide triggers the proliferation pathways in tumor cells but not in normal cells. Based on the early success of IL-24 as a potential anticancer therapy in patients (26), we are optimistic that, with the appropriate modifications, this cytokine may become a frontline therapeutic agent for the treatment of multiple human cancers.

\section{Acknowledgements}

The present study was supported by the Beijing Natural Science Foundation (grant no. 7142117) to Q.M., the National Basic Research Program of China (973 Program, grant no. 2009CB521704) to Z.C., the Person Project of Beijing Jiaotong University (grant no. KSRC12003532) and the Beijing Cancer Rehabilitation Association Foundation (grant no. KSM13001531) to Z.W., the National Natural Science Foundation of China (grant no. 81071855) and the Person Project of Beijing Jiaotong University (grant no. KSRC11004536) to H.J.

\section{References}

1. Vogelstein B and Kinzler KW: Cancer genes and the pathways they control. Nat Med 10: 789-799, 2004. 
2. Janet $\mathrm{P}$, Whitney $\mathrm{S}$, Fang X, et al: Rational design of shepherdin, a novel anticancer agent. Cancer Cell 7: 457-468, 2005.

3. Jiang H, Lin JJ, Su ZZ, et al: Subtraction hybridization identifies a novel melanoma differentiation associated gene, mda-7, modulated during human melanoma differentiation, growth and progression. Oncogene 11: 2477-2286, 1995.

4. Gupta P, Su ZZ, Lebedeva IV, et al: mda-7/IL-24: multifunctional cancer-specific apoptosis-inducing cytokine. Pharmacol Ther 111: 596-628, 2006

5. Mhashilkar AM, Schrock RD, Hindi M, et al: Melanoma differentiation associated gene-7 (mda-7): a novel anti-tumor gene for cancer gene therapy. Mol Med 7: 271-282, 2001.

6. Dent P, Yacoub A, Hamed HA, et al: MDA-7/IL-24 as a cancer therapeutic: from bench to bedside. Anticancer Drugs 21: 725-731, 2010.

7. Caudell EG, Mumm JB, Poindexter N, et al: The protein product of the tumor suppressor gene, melanoma differentiationassociated gene 7, exhibits immunostimulatory activity and is designated IL-24. J Immunol 168: 6041-6046, 2002.

8. Mumm JB, Ekmekcioglu S, Poindexter NJ, et al: Soluble human MDA-7/IL-24: characterization of the molecular form(s) inhibiting tumor growth and stimulating monocytes. J Interferon Cytokine Res 26: 877-886, 2006.

9. Su ZZ, Lebedeva IV, Sarkar D, et al: Melanoma differentiation associated gene-7, mda-7/IL-24, selectively induces growth suppression, apoptosis and radiosensitization in malignant gliomas in a p53-independent manner. Oncogene 22: 1164-1180, 2003.

10. Su Z, Emdad L, Sauane M, et al: Unique aspects of mda-7/IL-24 antitumor bystander activity: establishing a role for secretion of MDA-7/IL- 24 protein by normal cells. Oncogene 24: 7552-7566, 2005.

11. Yacoub A, Liu R, Park MA, et al: Cisplatin enhances protein kinase R-like endoplasmic reticulum kinase- and CD95-dependent melanoma differentiation-associated gene-7/interleukin-24induced killing in ovarian carcinoma cells. Mol Pharmacol 77: 298-310, 2010.

12. Lebedeva IV, Washington I, Sarkar D, et al: Strategy for reversing resistance to a single anticancer agent in human prostate and pancreatic carcinomas. Proc Natl Acad Sci USA 104: 3484-3489, 2007.

13. Bocangel D, Zheng M, Mhashilkar A, et al: Combinatorial synergy induced by adenoviral-mediated mda-7 and Herceptin in Her- $2^{+}$breast cancer cells. Cancer Gene Ther 13: 958-968, 2006.

14. Su Z, Lebedeva IV, Gopalkrishnan RV, et al: A combinatorial approach for selectively inducing programmed cell death in human pancreatic cancer cells. Proc Natl Acad Sci USA 98: 10332-10337, 2001.

15. Lebedeva IV, Su ZZ, Sarkar D, et al: Melanoma differentiation associated gene-7, mda-7/interleukin-24, induces apoptosis in prostate cancer cells by promoting mitochondrial dysfunction and inducing reactive oxygen species. Cancer Res 63: 8138-8144, 2003.

16. Zerbini LF, Czibere A, Wang Y, et al: A novel pathway involving melanoma differentiation associated gene-7/interleukin-24 mediates nonsteroidal anti-inflammatory drug-induced apoptosis and growth arrest of cancer cells. Cancer Res 66: 11922-11931, 2006.

17. Chada S, Mhashilkar AM, Liu Y, et al: mda-7 gene transfer sensitizes breast carcinoma cells to chemotherapy, biologic therapies and radiotherapy: correlation with expression of bcl-2 family members. Cancer Gene Ther 13: 490-502, 2006.

18. Zerbini LF, Tamura RE, Correa RG, et al: Combinatorial effect of non-steroidal anti-inflammatory drugs and NF- $\kappa \mathrm{B}$ inhibitors in ovarian cancer therapy. PLoS One 6: e24285, 2011.

19. Fisher PB: Is mda-7/IL-24 a 'magic bullet' for cancer? Cancer Res 65: 10128-10138, 2005 .
20. Dong CY, Zhang F, Duan YJ, et al: mda-7/IL-24 inhibits the proliferation of hematopoietic malignancies in vitro and in vivo. Exp Hematol 36: 938-946, 2008.

21. Saeki T, Mhashilkar A, Swanson X, et al: Inhibition of human lung cancer growth following adenovirus-mediated mda-7 gene expression in vivo. Oncogene 21: 4558-4566, 2002.

22. Tamai H, Miyake K, Yamaguchi H, et al: AAV8 vector expressing IL24 efficiently suppresses tumor growth mediated by specific mechanisms in MLL/AF4-positive ALL model mice. Blood 11: 64-71, 2012.

23. Pataer A, Hu W, Xiaolin L, et al: Adenoviral endoplasmic reticulum-targeted mda-7/interleukin-24 vector enhances human cancer cell killing. Mol Cancer Ther 7: 2528-2535, 2008.

24. Sauane M, Su ZZ, Gupta P, et al: Autocrine regulation of mda-7/ IL-24 mediates cancer-specific apoptosis. Proc Natl Acad Sci USA 105: 9763-9768, 2008.

25. Margue C and Kreis S: IL-24: physiological and supraphysiological effects on normal and malignant cells. Curr Med Chem 17: 3318-3326, 2010

26. Dash R, Bhutia SK, Azab B, et al: mda-7/IL-24: a unique member of the IL-10 gene family promoting cancer-targeted toxicity. Cytokine Growth Factor Rev 21: 381-391, 2010.

27. Zheng M, Bocangel D, Ramesh R, et al: Interleukin-24 overcomes temozolomide resistance and enhances cell death by down-regulation of $O^{6}$-methylguanine-DNA methyltransferase in human melanoma cells. Mol Cancer Ther 7: 3842-3851, 2008.

28. Gupta P, Walter MR, Su ZZ, et al: BiP/GRP78 is an intracellular target for MDA-7/IL-24 induction of cancer-specific apoptosis. Cancer Res 66: 8182-8191, 2006.

29. Gupta P, Emdad L, Lebedeva IV, et al: Targeted combinatorial therapy of non-small cell lung carcinoma using a GST-fusion protein of full-length or truncated MDA-7/IL-24 with Tarceva. J Cell Physiol 215: 827-836, 2008.

30. Rosal R, Brandt-Rauf P, Pincus MR, et al: The role of alphahelical structure in p53 peptides as a determinant for their mechanism of cell death: necrosis versus apoptosis. Adv Drug Deliv Rev 57: 653-660, 2005

31. Böttger A, Böttger V, Sparks A, et al: Design of a synthetic Mdm2-binding mini protein that activates the p53 response in vivo. Curr Biol 7: 860-869, 1997.

32. Tong AW, Nemunaitis J, Su D, et al: Intratumoral injection of INGN 241, a nonreplicating adenovector expressing the melanomadifferentiation associated gene-7 (mda-7/IL24): biologic outcome in advanced cancer patients. Mol Ther 11: 160-172, 2005.

33. Cunningham CC, Chada S, Merritt JA, et al: Clinical and local biological effects of an intratumoral injection of mda-7 (IL24; INGN 241) in patients with advanced carcinoma: a phase I study. Mol Ther 11: 149-159, 2005.

34. Dent P, Yacoub A, Hamed HA, et al: The development of MDA-7/ IL-24 as a cancer therapeutic. Pharmacol Ther 128: 375-384, 2010.

35. Sauane M, Gopalkrishnan RV, Sarkar D, et al: MDA-7/IL-24: novel cancer growth suppressing and apoptosis inducing cytokine. Cytokine Growth Factor Rev 14: 35-51, 2003.

36. Chada S, Sutton RB, Ekmekcioglu S, et al: MDA-7/IL-24 is a unique cytokine - tumor suppressor in the IL-10 family. Int Immunopharmacol 4: 649-667, 2004.

37. Kotenko SV: The family of IL-10-related cytokines and their receptors: related, but to what extent? Cytokine Growth Factor Rev 13: 223-240, 2002.

38. Lebedeva IV, Emdad L, Su ZZ, et al: mda-7/IL-24, novel anticancer cytokine: Focus on bystander antitumor, radiosensitization and antiangiogenic properties and overview of the phase I clinical experience (Review). Int J Oncol 31: 985-1007, 2007.

39. Chada S, Mhashilkar AM, Ramesh R, et al: Bystander activity of Ad-mda7: human MDA-7 protein kills melanoma cells via an IL-20 receptor-dependent but STAT3-independent mechanism. Mol Ther 10: 1085-1095, 2004. 